\title{
Effect of ambroxol on the concentration of cefotaxime in the bronchoalveolar lavage fluid of rats with pulmonary fibrosis
}

\author{
FENG CHEN, YUAN-XIA ZHANG and CAI-QING ZHANG \\ Department of Respiratory Medicine, Shandong Provincial Qianfoshan Hospital, Shandong University, \\ Jinan, Shandong 250014, P.R. China
}

Received March 20,2014; Accepted October 2, 2014

DOI: 10.3892/etm.2014.2112

\begin{abstract}
This study aimed to investigate the effect of ambroxol on the concentration of cefotaxime in the bronchioalveolar lavage fluid of rats with bleomycin-induced pulmonary fibrosis. A total of $54 \mathrm{Wistar}$ male rats were randomly divided into three groups, namely the normal control group, model group and ambroxol group. On experimental day 0 , the rats were intratracheally instilled with bleomycin $(5 \mathrm{mg} / \mathrm{kg}$ body weight) or sterile saline. The rats in the ambroxol group were then treated with ambroxol ( $35 \mathrm{mg} / \mathrm{kg} /$ day) intraperitoneally. On days 7, 14, 28 after instillation, six rats from each group were sacrificed, bronchial alveolar fluids were recovered and the lungs were collected for histopathological examination following the injection of cefotaxime $(600 \mathrm{mg} / \mathrm{kg})$ intravenously. The concentration of cefotaxime in the bronchial alveolar fluids was assayed by a liquid chromatography-mass spectrometry method. On day 7 , the concentration of cefotaxime in the bronchial alveolar fluid of the ambroxol group was lower than that of the model group. On day 14, the concentration of cefotaxime in the bronchial alveolar fluids of the ambroxol group was higher than that of the model group, and the difference between these groups was significant statistically $(\mathrm{P}<0.001)$. On day 28 , the concentration of cefotaxime in the bronchial alveolar fluids of the ambroxol group decreased sharply, and was lower than that of the model group $(\mathrm{P}=0.126)$. These results indicate that ambroxol increased the concentration of cefotaxime in the bronchial alveolar fluids at the primary fibrosis stage.
\end{abstract}

\section{Introduction}

Idiopathic pulmonary fibrosis (IPF) is the most common specific form of idiopathic interstitial pneumonia. It is a chronic, progressive and irreversible lung disease with

Correspondence to: Dr Cai-Qing Zhang, Department of Respiratory Medicine, Shandong Provincial Qianfoshan Hospital, Shandong University, 16766 Jingshi Road, Jinan, Shandong 250014, P.R. China E-mail:1gbkcn@163.com

Key words: pulmonary fibrosis, ambroxol, cefotaxime unknown cause and is usually fatal. Its histopathological or radiological appearance is similar to that typically observed for usual interstitial pneumonia (1-3). The mean survival time for diagnosed patients is 2.5 to 3.5 years (4); the survival period is short and the mortality rate is high. Ischemic heart disease, heart failure, bronchial cancer, infection and pulmonary embolism are significant causes of mortality in patients with IPF (5-8).

IPF complicated by infection can cause respiratory function to decline (4), which may lead to exacerbations and further reduce the arterial oxygen pressure. Active control of infection should help to improve lung function and reduce mortality.

The success of antibiotic therapy not only depends on the sensitivity of pathogenic microorganisms, but also the drug concentration in infected tissues, which is particularly important in the treatment of respiratory tract infections (9). Therefore, improving the concentration of antimicrobial drugs in the bronchial-pulmonary tissues is likely to help in controlling infection. According to previous studies, ambroxol increases the concentration of amoxicillin, erythromycin, ampicillin and other antibacterial drugs in the normal lung tissues of animals and humans (9-11). If ambroxol is able to improve antimicrobial concentrations in the lung tissue of patients with pulmonary fibrosis, treatment efficacy may be improved. However, to the best of our knowledge, whether ambroxol has an impact on the antimicrobial drug concentration in pulmonary interstitial fibrosis has not previously been reported. Bronchoalveolar lavage techniques are suitable for detecting the concentrations of antimicrobial agents in lung tissues (10). In the present study, the impact of ambroxol on the concentration of cefotaxime in the lung tissue of rats with pulmonary fibrosis was tested by a bronchoalveolar lavage technique to evaluate the role of ambroxol.

\section{Materials and methods}

Animal grouping and model preparation. A total of 54 male Wistar rats (clean grade) with weights of 180-220 g were purchased from the Animal Center of Shandong University (Jinan, China). The animals were randomly divided into three groups. These were a normal control group (group A), model group (group B) and ambroxol hydrochloride group (group C). The rats of the $\mathrm{B}$ and $\mathrm{C}$ groups were administered an intratracheal injection of bleomycin $(5 \mathrm{mg} / \mathrm{kg})$, and those of group A 
were administered an infusion of saline via the trachea. In addition, a daily intraperitoneal injection of ambroxol hydrochloride $35 \mathrm{mg} / \mathrm{kg}$ ( $7 \mathrm{mg}$ for each rat) (Hebei Aierhaitai Pharmaceutical Co., Ltd., Shijiazhuang, China) was administered to the rats in group C. This study was carried out in strict accordance with the recommendations in the Guide for the Care and Use of Laboratory Animals (8th edition, 2011) of the National Institutes of Health. The animal use protocol was reviewed and approved by the Institutional Animal Care and Use Committee (IACUC) of Shandong Provincial Qianfoshan Hospital (Permit Number: 20060828001). On days 7, 14, 28 after the perfusion of bleomycin, six rats were collected from each group to obtain bronchoalveolar lavage fluid following the injection of cefotaxime sodium $(600 \mathrm{mg} / \mathrm{kg}$; Suzhou Dawnrays Pharmaceutical Co., Ltd., Suzhou, China) via the tail vein. Liquid chromatography-mass spectrometry (MS) was used to determine the concentration of cefotaxime in the lavage fluid. Lung tissues were collected for pathological observation.

Specimen collecting and handling. A $10 \%$ chloral hydrate solution was used to anesthetize the rats by intraperitoneal injection. Following fixation, the trachea was isolated from the neck, the trachea was cut, a catheter was inserted and ligation with silk, and $2 \mathrm{ml}$ saline was injected into the catheter. Following twice repeated washing, bronchoalveolar lavage fluid was collected in test tubes and centrifuged at $444 \mathrm{x} g$ for $10 \mathrm{~min}$. The supernatant was stored in an EP tube at $-20^{\circ} \mathrm{C}$. Lungs were removed and fixed with $10 \%$ formaldehyde solution until pathological examination one week later.

Pathological examination. The right lung was dehydrated, rendered transparent and embedded in wax. The 7- $\mu \mathrm{m}$ sections were stained with hematoxylin and eosin and the pathological changes were observed under a light microscope. The extent of alveolitis and pulmonary fibrosis was evaluated according to the method of Szapiel (12). Grading standards: (-), no alveolitis or pulmonary fibrosis; (+), mild alveolitis or pulmonary fibrosis with an affected area of $<20 \%$ of the whole lung; $(++)$ moderate alveolitis or pulmonary fibrosis accounting for $20-50 \%$ of the lung; and (+++), severe alveolitis or pulmonary fibrosis with an affected area of $>50 \%$.

Measurement of cefotaxime concentration. Chromatography was conducted using a Zorbax SB-C18 analytical column (150x4.6 mm; internal diameter, $5 \mu \mathrm{m}$ ) from Agilent Technologies (Santa Clara, CA, USA) and a $0.45-\mu \mathrm{m}$ line filter (Agilent Technologies). The mobile phase comprised methanol and water (containing $0.1 \%$ acetic acid) in a $30: 70$ ratio by volume; the flow rate was $1.0 \mathrm{ml} / \mathrm{min}$ and was split to provide a flow rate of $\sim 0.3 \mathrm{ml} / \mathrm{min}$ into a mass spectrometer (Agilent1100Trap VL-ion trap, Agilent Technologies), with an injection volume of $5 \mu 1$.

MS was conducted using an electrospray ionization (ESI) source, a heated capillary temperature of $350^{\circ} \mathrm{C}$, a spray gas $\left(\mathrm{N}_{2}\right)$ pressure of $30 \mathrm{psi}$ and a drying gas $\left(\mathrm{N}_{2}\right)$ flow of $10 \mathrm{l} / \mathrm{min}$. The positive ion detection mode and multiple scanning with the multiple reaction monitoring (MRM) mode were used. The ionic reaction $\mathrm{m} / \mathrm{z} 500 \rightarrow 440$ was quantitatively analyzed at a fragmentation voltage of $0.8 \mathrm{~V}$.

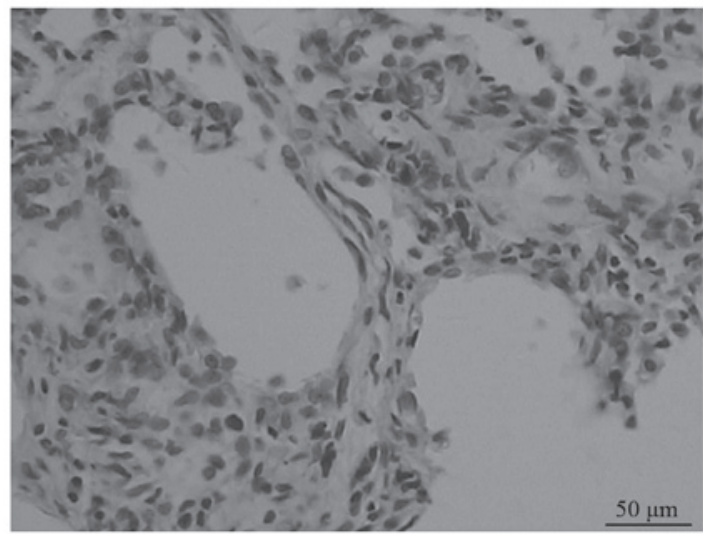

Figure 1. Microscopic examination of the 28-day model group (hematoxylin and eosin staining; magnification, $\mathrm{x} 400$ ).

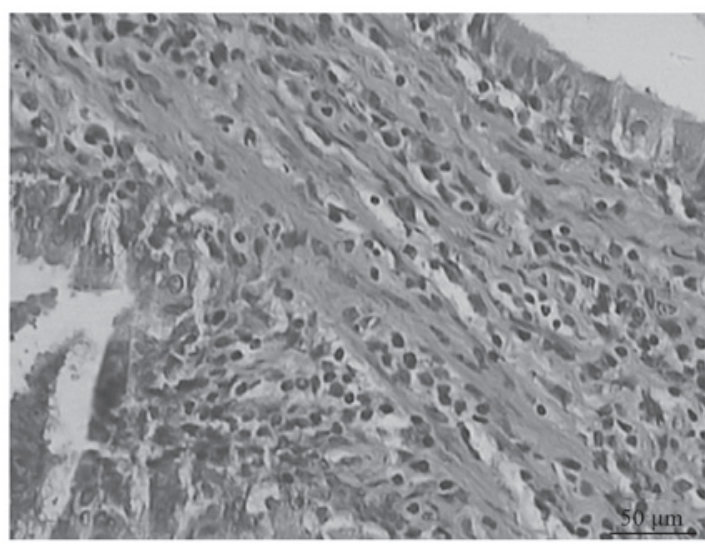

Figure 2. Microscopic examination of the 28-day ambroxol-treated group (hematoxylin and eosin staining; magnification, $\mathrm{x} 400$ ).

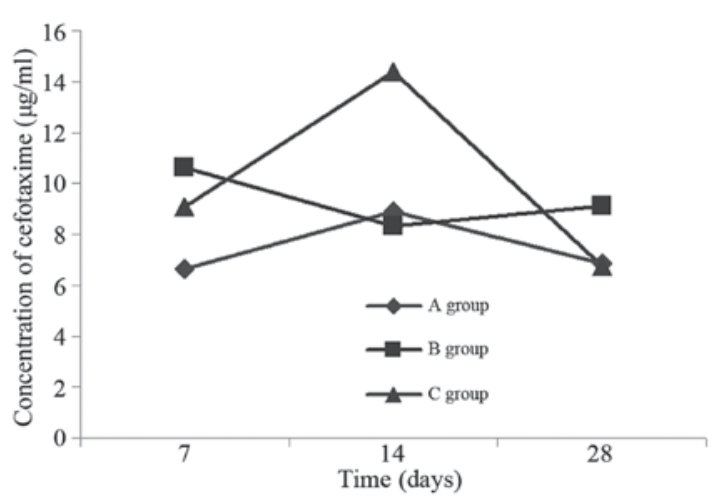

Figure 3. Line chart of the cefotaxime concentration in the bronchoalveolar lavage fluid at different time points in the three groups of rats.

Samples were prepared by placing $100 \mu \mathrm{l}$ bronchoalveolar lavage fluid in a $5 \mathrm{ml}$ plastic centrifuge tube, adding $400 \mu \mathrm{l}$ methanol, vortexing for $0.5 \mathrm{~min}$ and centrifuging at $1,776 \mathrm{x} \mathrm{g}$ for $10 \mathrm{~min}$. Then, $5 \mu \mathrm{l}$ supernatant was used for analysis.

The specificity of the method was determined using a $10 \mu \mathrm{g} / \mathrm{ml}$ methanolic solution of cefotaxime sodium as the control. An ESI source was used, with a heated capillary temperature of $325^{\circ} \mathrm{C}$, spray gas $\left(\mathrm{N}_{2}\right)$ pressure of $10 \mathrm{psi}$ and drying gas $\left(\mathrm{N}_{2}\right)$ flow of $4 \mathrm{l} / \mathrm{min}$. The perfusion injection mode 
Table I. Cefotaxime concentration in the bronchoalveolar lavage fluid at different time points in the three groups of rats $(\mu \mathrm{g} / \mathrm{ml})$.

\begin{tabular}{lrrr}
\hline Group & \multicolumn{1}{c}{ Day 7} & Day 14 & Day 28 \\
\hline A & $6.64 \pm 0.32$ & $8.90 \pm 2.48$ & $6.85 \pm 1.08$ \\
B & $10.63 \pm 2.19$ & $8.33 \pm 2.34$ & $9.11 \pm 4.11$ \\
C & $9.09 \pm 2.14$ & $14.39 \pm 3.21$ & $6.74 \pm 1.63$ \\
\hline
\end{tabular}

Table II. Pairwise comparison of cefotaxime concentration in the bronchoalveolar lavage fluid between groups on day 7.

\begin{tabular}{|c|c|c|c|c|c|c|}
\hline \multirow[b]{2}{*}{ (I) Group } & \multirow[b]{2}{*}{ (J) Group } & \multirow{2}{*}{$\begin{array}{c}\text { Mean } \\
\text { range (I-J) }\end{array}$} & \multirow{2}{*}{$\begin{array}{l}\text { Standard } \\
\text { error }\end{array}$} & \multirow{2}{*}{$\begin{array}{c}\text { Significant } \\
\text { level }\end{array}$} & \multicolumn{2}{|c|}{$95 \%$ credibility interval } \\
\hline & & & & & Lowest limit & Highest limit \\
\hline \multirow[t]{2}{*}{ Group B } & Group A & 3.9896 & 1.070 & 0.001 & 1.7860 & 6.1932 \\
\hline & Group C & 1.5331 & 1.070 & 0.164 & -0.6705 & 3.7368 \\
\hline
\end{tabular}

Table III. Pairwise comparison of cefotaxime concentration in the bronchoalveolar lavage fluid between groups on day 14.

\begin{tabular}{llccccc}
\hline & & & & & \multicolumn{2}{c}{$95 \%$ credibility interval } \\
\cline { 3 - 6 } (I) Group & $(\mathrm{J})$ Group & $\begin{array}{c}\text { Mean } \\
\text { range (I-J) }\end{array}$ & $\begin{array}{c}\text { Standard } \\
\text { error }\end{array}$ & $\begin{array}{c}\text { Significant } \\
\text { level }\end{array}$ & Lowest limit & Highest limit \\
\hline \multirow{2}{*}{ Group B } & Group A & -0.5794 & 1.479 & 0.698 & -3.6249 & -9.1042 \\
& Group C & -6.0588 & 1.479 & 0.000 & -3.0133 \\
\hline
\end{tabular}

Table IV. Pairwise comparison of cefotaxime concentration in the bronchoalveolar lavage fluid between groups on day 28.

\begin{tabular}{|c|c|c|c|c|c|c|}
\hline \multirow[b]{2}{*}{ (I) Group } & \multirow[b]{2}{*}{ (J) Group } & \multirow{2}{*}{$\begin{array}{c}\text { Mean } \\
\text { range }(\mathrm{I}-\mathrm{J})\end{array}$} & \multirow{2}{*}{$\begin{array}{l}\text { Standard } \\
\text { error }\end{array}$} & \multirow{2}{*}{$\begin{array}{c}\text { Significant } \\
\text { level }\end{array}$} & \multicolumn{2}{|c|}{$95 \%$ credibility interval } \\
\hline & & & & & Lowest limit & Highest limit \\
\hline \multirow[t]{2}{*}{ Group B } & Group A & 2.2565 & 1.498 & 0.145 & -0.8290 & 5.3421 \\
\hline & Group C & 2.3720 & 1.498 & 0.126 & -0.7136 & 5.4576 \\
\hline
\end{tabular}

was used, with a flow rate of $5 \mu \mathrm{l} / \mathrm{min}$. Cefotaxime sodium mainly generated $[\mathrm{M}+\mathrm{Na}]^{+}(\mathrm{m} / \mathrm{z} 500)$ in the positive ion mode. Selective ion scanning was used to analyze the $[\mathrm{M}+\mathrm{Na}]^{+}$peaks by fragmentation product. The most commonly observed ion fragment of cefotaxime sodium, which had an $\mathrm{m} / \mathrm{z}$ ratio of 440 , was considered as the product ion in the quantitative analysis.

A standard curve was generated by taking a precise amount $(1.0 \mathrm{mg} / \mathrm{ml})$ of cefotaxime sodium and adding methanol to prepare solutions of different concentrations $(5,10$ and $50 \mu \mathrm{g} / \mathrm{ml})$. A 5- $\mu 1$ aliquot of the sample solution was subjected to analysis by MS. The concentration was plotted as the abscissa and the peak area as the vertical axis of the linear standard curve to prepare a standard quantitative curve (in units of $\mu \mathrm{g} / \mathrm{ml}$ ).

Statistical analysis. The results are expressed as mean \pm standard deviation, using single-factor analysis of variance (one-way ANOVA) to analyze homogeneity of variance,
ANOVA and pairwise comparisons. Statistical analysis was performed using SPSS statistical software, version 13.0 (SPSS, Inc., Chicago, IL, USA).

\section{Results}

Morphological changes. On day 7, under a light microscope, the model group displayed moderate to severe alveolitis, but only mild alveolitis was observed on day 28. Alveolitis of the ambroxol group was mild compared with that of the untreated group. Fibrosis began from day 14 in the model group, and progressively developed until day 28 , when it reached a peak (Fig. 1). The extent of pulmonary fibrosis in the ambroxol group was reduced compared with that in the model group (Fig. 2).

Cefotaxime concentration. The cefotaxime concentration in the bronchoalveolar lavage fluid of the model group was 
higher than that in the normal control on day 7, was reduced to a minimum on day 14 , and then rose again by day 28 (Table I, Fig. 3). On day 7, the cefotaxime concentration in the bronchoalveolar lavage fluid of the ambroxol group was reduced in comparison with that in the model group; however, the difference was not statistically significant $(\mathrm{P}=0.164$; Table II). On day 14, the cefotaxime concentration in the bronchoalveolar lavage fluid of the ambroxol group rose and was higher than that of the model group; the difference between the ambroxol and model groups was statistically significant $(\mathrm{P}<0.001$; Table III). On day 28, the concentration in the ambroxol group dropped sharply, and was lower than that in the model group; however, the difference was not statistically significant $(\mathrm{P}=0.126$; Table IV).

\section{Discussion}

IPF complicated by infection can cause respiratory function to decline (4), exacerbate disease and increase mortality. One of the factors that is key to the success of anti-infective therapy is the concentration of antimicrobial agent in the infected tissues (9). Improving the lung tissue concentrations of antimicrobial drugs should help to control infection and improve the prognosis.

The present study aimed to determine the impact of ambroxol on the antimicrobial drug concentration in the lung tissues of rats with pulmonary fibrosis. Cephalosporins are a commonly used class of antibacterial drugs, which enter the lung tissue by diffusion mechanisms (13). In the present study, cefotaxime sodium was selected as a representative of this drug class. It was found that the concentration of cefotaxime in the bronchoalveolar lavage fluid of the model group on day 7 (the alveolitis period) was significantly higher than that of the control group due to an increase of vascular permeability in the alveolitis period in the model group, which is consistent with the literature (14). The concentration in the model group had decreased by day 14 (the initial fibrosis period) and rose again on day 28 (the fibrosis stage), when no significant difference was detected when compared with the control group.

It was observed that the salt of ambroxol, in addition to improving the concentration of cefotaxime in the bronchoalveolar lavage fluid of the early fibrosis period, also reduced fibrosis, which is consistent with the literature $(15,16)$. Previous studies have demonstrated that ambroxol has good anti-inflammatory and antioxidant effects (17-20), and can inhibit the synthesis and release of cytokines and inflammatory mediators (21). Oxygen free radical damage is an important aspect of interstitial pulmonary fibrosis. In the present study, ambroxol hydrochloride reduced alveolitis and pulmonary fibrosis. In the alveolitis and fibrosis periods, the cefotaxime sodium concentration in the bronchoalveolar lavage fluid of the ambroxol group was not significantly different from that of the model group, but was higher than that of the model group in the initial fibrosis period, suggesting that the increased impact of ambroxol hydrochloride on the cefotaxime sodium concentration in the bronchoalveolar lavage fluid in the early fibrosis period had no significant association with its anti-inflammatory and antifibrotic effects.

In conclusion, the results revealed that ambroxol hydrochloride can improve the cefotaxime sodium concentration in bronchoalveolar lavage fluid. Thus, ambroxol may improve anti-infection treatment in the early fibrosis period, and these results may act as a reference for clinical anti-infection treatment. The mechanism by which ambroxol hydrochloride increases the concentration of cefotaxime sodium in bronchoalveolar lavage fluid requires further study.

\section{References}

1. American Thoracic Society and European Respiratory Society: Idiopathic pulmonary fibrosis: Diagnosis and treatment. International consensus statement. Am J Respir Crit Care Med 161: 646-664, 2000.

2. American Thoracic Society and European Respiratory Society: International multidisciplinary consensus classification of the idiopathic interstitial pneumonias. Am J Respir Crit Care Med 165: 277-304, 2002.

3. Raghu G, Collard HR, Egan JJ, et al; ATS/ERS/JRS/ALAT Committee on Idiopathic Pulmonary Fibrosis: An official ATS/ERS/JRS/ALAT statement: Idiopathic pulmonary fibrosis: evidence-based guidelines for diagnosis and management. Am J Respir Crit Care Med 183: 788-824, 2011.

4. Ley B, Collard HR and King TE Jr: Clinical course and prediction of survival in idiopathic pulmonary fibrosis. Am J Respir Crit Care Med 183: 431-440, 2011.

5. Martinez FJ, Safrin S, Weycker D, et al; IPF Study Group: The clinical course of patients with idiopathic pulmonary fibrosis. Ann Intern Med 142: 963-967, 2005.

6. Olson AL, Swigris JJ, Lezotte DC, Norris JM, Wilson CG and Brown KK: Mortality from pulmonary fibrosis increased in the United States from 1992 to 2003. Am J Respir Crit Care Med 176: 277-284, 2007.

7. King TE Jr, Albera C, Bradford WZ, et al; INSPIRE Study Group: Effect of interferon gamma-1b on survival in patients with idiopathic pulmonary fibrosis (INSPIRE): a multicentre, randomised, placebo-controlled trial. Lancet 374: 222-228, 2009.

8. Hubbard RB, Smith C, Le Jeune I, Gribbin J and Fogarty AW: The association between idiopathic pulmonary fibrosis and vascular disease: a population-based study. Am J Respir Crit Care Med 178: 1257-1261, 2008.

9. Spátola J, Poderoso JJ, Wiemeyer JC, et al: Influence of ambroxol on lung tissue penetration of amoxillin. Arzneimittelforschung 37: 965-966, 1987.

10. Gené R, Poderoso JJ, Corazza C, Lasala MB, Wiemeyer JC, Fernández $\mathrm{M}$ and Guerreiro RB: Influence of ambroxol on amoxicillin levels in bronchoalveolar lavage fluid. Arzneimittelforschung 37: 967-968, 1987.

11. Wiemeyer JC: Influence of ambroxol on the bronchopulmonary level of antibiotics. Arzneimittelforschung 31: 974-976, 1981.

12. Szapiel SV, Elson NA, Fulmer JD, Hunninghake GW and Crystal RG. Bleomycin-induced interstitial pulmonary disease in the nude, athymic mouse. Am Rev Respir Dis 120: 893-899, 1979.

13. Wang F. Respiratory Tract Infection. Infectious diseases and antimicrobial therapy. 2nd edition, Shanghai Medical University Publishing House, Shanghai, China, pp 216-223, 2000.

14. Morgan EJ and Petty TL: Summary of the National Mucolytic Study. Chest 97 (2 Suppl): 24S-27S, 1990.

15. Zhi QM, Yang LT and Sun HC: Protective effect of ambroxol against paraquat-induced pulmonary fibrosis in rats. Intern Med 50: 1879-1887, 2011

16. Pozzi E, Salmona M, Masturzo P, Genghini M, Scelsi M, Spialtini L and Luisetti M: Role of alveolar phospholipids in bleomycin-induced pulmonary fibrosis in the rat. Respiration 51: 23-32, 1987.

17. Winsel K: The antioxidative and inflammation inhibiting properties of ambroxol. Pneumologie 46: 461-475, 1992 (In German).

18. Beeh KM, Beier J, Esperester A and Paul LD: Antiinflammatory properties of ambroxol. Eur J Med Res 13: 557-562, 2008.

19. Nowak D, Antczak A, Król M, et al: Antioxidant properties of Ambroxol. Free Radic Biol Med 16: 517-522, 1994.

20. Piotrowski WJ, Pietras T, Kurmanowska Z, et al: Effect of paraquat intoxication and ambroxol treatment on hydrogen peroxide production and lipid peroxidation in selected organs of rat. J Appl Toxicol 16: 501-507, 1996.

21. Stockley RA, Shaw J and Burnett D: Effect of ambroxol of neutrophil chemotaxis in vitro. Agents Actions 24: 292-296, 1988. 